\title{
Gegenbauer Polynomial Method for Linear Complex Differential Equations
}

\author{
Faruk DÜȘ̈NCELi் ${ }^{1}$, Ercan ÇELIK ${ }^{2}$ \\ 1. Faculty of Economic and Administrative Sciences, Mardin Artuklu University, Mardin, Turkey \\ 2. Faculty of Science, Ataturk University, Erzurum, Turkey \\ farukdusunceli@artuklu.edu.tr, ercelik@atauni.edu.tr
}

\begin{abstract}
In this paper, gegenbauer polynomials was used to get numerical solution of linear complex differential equations. The equations in the form of matrix polynomial were occurred.
\end{abstract}

Keywords: Linear complex differential equations; gegenbauer polynomial; collocation method; numerical solution.

\section{Introduction}

Different type differential equations solved with orthogonal polynomials[1-6]. In this paper, the matrix relations between the gegenbauer polynomials and their derivatives, we develop a method called the gegenbauer collocation method for solving linear complex differential equation.

$$
\sum_{n=0}^{m} P_{n}(z) f^{(n)}(z)=g(z)
$$

with the initial conditions

$$
f^{(t)}(\alpha)=\vartheta_{t} \quad t=0,1, \ldots, m-1
$$

We will let $f(z)$ is unknown function, $P_{n}(z)$ and $g(z)$ are analytical functions in the circular domain which $D=\left\{z=x+i y, z \in C,\left|z-z_{0}\right| \leq r, r \in R^{+}\right\}, ; \alpha, z_{0} \in D, \vartheta_{t}$ is appropriate complex or real constant.

We assume that the solution of (1) under the conditions (2) is approximated in the form

$$
f(z)=\sum_{n=0}^{N} a_{n} Q_{n}(z), z \in D
$$

which is the gegenbauer series of the unknown function $f(z)$, where all of $a_{n}$ are the gegenbauer coefficients to be determined. We also use the collocation points

$$
z_{p p}=z_{0}+\frac{r}{N} p e^{\frac{i \theta}{N} p}, 0<\theta \leq 2 \pi, r \in R^{+}, p \in 0,1, \ldots, N
$$

\section{2 ) Main Matrix Relations}

Gegenbauer polynomials defined as follows[7];

$$
\begin{gathered}
Q(0, a, x)=1 ; \\
Q(1, a, x)=2 a x ; \\
Q(n, a, x)=\frac{2 x(n+a-1)}{n} Q(n-1, a, x)-\frac{n+2 a-2}{n} Q(n-2, a, x)
\end{gathered}
$$


We can write the Gegenbauer polynomials in the matrix form as

$$
Q(z)=Z(z) M^{T}
$$

where

$$
Q(z)=\left[\begin{array}{llll}
Q_{0}(z) & Q_{1}(z) & \ldots & Q_{N}(z)
\end{array}\right]
$$

and

$$
Z(z)=\left[\begin{array}{lllll}
1 & Z & z^{2} & \ldots & z^{N}
\end{array}\right]
$$

On the other hand,

If $\mathrm{N}$ is odd,

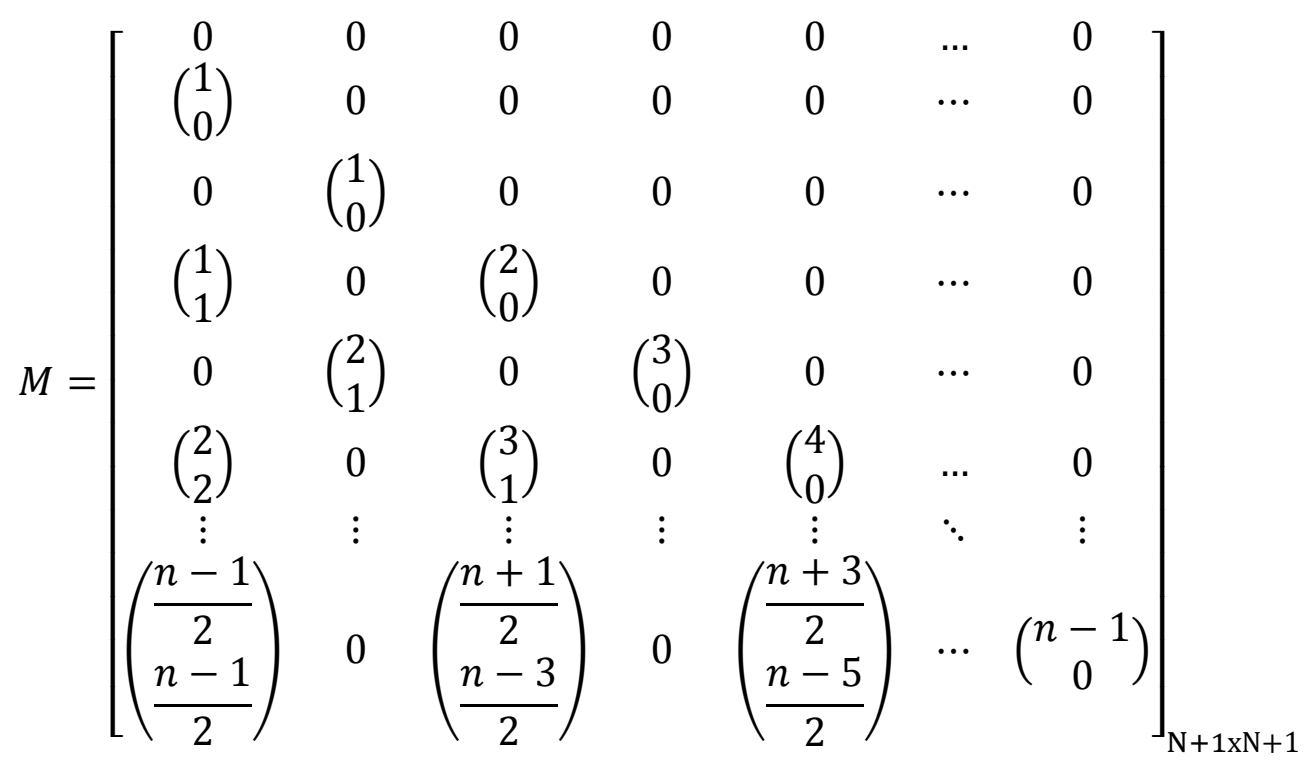

If $\mathrm{N}$ is even,

$$
M=\left[\begin{array}{ccccccc}
0 & 0 & 0 & 0 & 0 & \ldots & 0 \\
\left(\begin{array}{c}
1 \\
0
\end{array}\right) & 0 & 0 & 0 & 0 & \cdots & 0 \\
0 & \left(\begin{array}{l}
1 \\
0
\end{array}\right) & 0 & 0 & 0 & \ldots & 0 \\
\left(\begin{array}{c}
1 \\
1
\end{array}\right) & 0 & \left(\begin{array}{l}
2 \\
0
\end{array}\right) & 0 & 0 & \cdots & 0 \\
0 & \left(\begin{array}{c}
2 \\
1
\end{array}\right) & 0 & \left(\begin{array}{c}
3 \\
0
\end{array}\right) & 0 & \ldots & 0 \\
\left(\begin{array}{c}
2 \\
2
\end{array}\right) & 0 & \left(\begin{array}{c}
3 \\
1
\end{array}\right) & 0 & \left(\begin{array}{c}
4 \\
0
\end{array}\right) & \ldots & 0 \\
\vdots & \vdots & \vdots & \ddots & \vdots \\
0 & \left(\begin{array}{c}
\frac{n}{2} \\
\frac{n-2}{2}
\end{array}\right) & 0 & \left(\begin{array}{c}
\frac{n+2}{2} \\
\frac{n-3}{2}
\end{array}\right) & 0 & \cdots & \left(\begin{array}{c}
n-1 \\
0
\end{array}\right)
\end{array}\right]_{\mathrm{N}+1 \times \mathrm{N}+1}
$$


We consider the desired solution $f(z)$ of Eq. (3)

$$
f(z)=Q(z) A ; \quad A=\left[\begin{array}{llll}
a_{0} & a_{1} & \ldots & a_{N}
\end{array}\right]^{T}
$$

the relation between the matrix $Q(z)$ and its derivatives $Q^{\prime}(z), Q^{(2)}(z), \ldots, Q^{(n)}(z)$ are

$$
\begin{gathered}
Q^{\prime}(z)=Q(z) K^{T} \\
Q^{(2)}(z)=Q(z)\left(K^{T}\right)^{2} \\
\vdots \\
Q^{(n)}(z)=Q(z)\left(K^{T}\right)^{n}
\end{gathered}
$$

Where

$$
K=\left[\begin{array}{ccccccc}
0 & 0 & 0 & 0 & 0 & \ldots & 0 \\
2 a & 0 & 0 & 0 & 0 & \cdots & 0 \\
0 & 2 a+2 & 0 & 0 & 0 & \cdots & 0 \\
2 a & 0 & 2 a+4 & 0 & 0 & \cdots & 0 \\
0 & 2 a+2 & 0 & 2 a+6 & 0 & \cdots & 0 \\
0 & 0 & 2 a+4 & 0 & 2 a+8 & \ldots & 0 \\
\vdots & \vdots & \vdots & \vdots & \vdots & \ddots & \vdots \\
0 & 0 & 0 & 0 & 0 & \ldots & 0
\end{array}\right]_{\mathrm{N}+1 \mathrm{xN}+1}
$$

By using the relations (7) and (8) we obtain the relation

$$
f^{(n)}(z)=Q^{(n)}(z) K^{T} A=Q(z)\left(K^{T}\right)^{n} A
$$

By substituting the collocation points $z=z_{p p}$ into the relation (9), we get the following matrix equations

$$
f^{(n)}\left(z_{p p}\right)=Q\left(z_{p p}\right)\left(K^{T}\right)^{n}, p \in 0,1, \ldots, N
$$

For $p=0,1, \ldots, N$, we can write the relation (10)

$$
\begin{gathered}
f^{(n)}\left(z_{00}\right)=Q\left(z_{00}\right)\left(K^{T}\right)^{n} \\
f^{(n)}\left(z_{11}\right)=Q\left(z_{11}\right)\left(K^{T}\right)^{n} \\
\vdots \\
f^{(n)}\left(z_{N N}\right)=Q\left(z_{N N}\right)\left(K^{T}\right)^{n}
\end{gathered}
$$

where

$$
Q=\left[\begin{array}{c}
Q_{00} \\
Q_{11} \\
\vdots \\
Q_{N N}
\end{array}\right]=\left[\begin{array}{ccccc}
Q_{0}\left(z_{00}\right) & Q_{1}\left(z_{00}\right) & Q_{2}\left(z_{00}\right) & \cdots & Q_{N}\left(z_{00}\right) \\
Q_{0}\left(z_{11}\right) & Q_{1}\left(z_{11}\right) & Q_{2}\left(z_{11}\right) & \cdots & Q_{N}\left(z_{11}\right) \\
\vdots & \vdots & \vdots & \vdots & \vdots \\
Q_{0}\left(z_{N N}\right) & Q_{1}\left(z_{N N}\right) & Q_{2}\left(z_{N N}\right) & \cdots & Q_{N}\left(z_{N N}\right)
\end{array}\right]
$$

let us substitute the collocation points (4) into Equation (1), 


$$
\sum_{n=0}^{m} P_{n}\left(z_{p p}\right) f^{(n)}\left(z_{p p}\right)=g\left(z_{p p}\right)
$$

we obtain the fundamental matrix equation of the relations (9)-(11),

$$
\sum_{n=0}^{m} \sum_{p=0}^{N} P_{n} Q\left(z_{p p}\right)\left(K^{T}\right)^{n} \mathrm{~A}=\sum_{p=0}^{N} G_{p}
$$

where

$P_{n}=\left[\begin{array}{cccc}P_{n}\left(z_{00}\right) & 0 & \cdots & 0 \\ 0 & P_{n}\left(z_{11}\right) & \cdots & 0 \\ \vdots & \vdots & \vdots & \vdots \\ 0 & 0 & \cdots & P_{n}\left(z_{N N}\right)\end{array}\right] \quad$ and $\quad G_{p}=\left[\begin{array}{c}g\left(z_{00}\right) \\ g\left(z_{11}\right) \\ \vdots \\ g\left(z_{N N}\right)\end{array}\right]$

Since the A is unknown and should be determined, therefore, the matrix equation (12) could be rewritten in the following form:

$$
W . A=G \text { or }[W ; G]=\left[w_{p q} ; g_{p}\right] p, q=0,1, \ldots, N
$$

where,

$$
W=\sum_{n=0}^{m} \sum_{p=0}^{N} P_{n} Q\left(z_{p p}\right)\left(K^{T}\right)^{n} \text { and } A=\left[\begin{array}{llll}
a_{0} & a_{1} & \ldots & a_{N}
\end{array}\right]
$$

We now write the corresponding matrix form of the initial conditions (2) by the aid of (12),

$$
f^{(t)}(\alpha)=Q(\alpha)\left(K^{T}\right)^{t} A=\vartheta_{t} \quad t=0,1, \ldots, m-1
$$

In other words the matrix form of the initial conditions could be rewritten as

where

$$
U_{t} A=\vartheta_{t} \quad t=0,1, \ldots, m-1
$$

$$
U_{t}=Q(\alpha)\left(K^{T}\right)^{t} \quad t=0,1, \ldots, m-1
$$

the augmented form of these equations is

$$
\left[U_{t} ; \vartheta_{t}\right]=\left[u_{t 0}, u_{t 1}, \ldots, u_{t N} ; \vartheta_{t}\right] \quad t=0,1, \ldots, m-1
$$

Finally, to find the unknown Gegenbauer coefficients $a_{n}, n=0,1, \ldots, N$, related with the approximate solution of the problem (1) under the initial conditions (2), we need to replace the $m$ rows of (14) by the last $m$ rows of the augmented matrix (13) and hence we have new augmented matrix

$$
[\widetilde{W} ; \tilde{G}]=\left[\begin{array}{cccccc}
w_{00} & w_{01} & \cdots & w_{0 N} & ; & g_{0} \\
w_{10} & w_{11} & \cdots & w_{1 N} & ; & g_{1} \\
\vdots & \vdots & \vdots & \vdots & \vdots & \vdots \\
w_{N-m 0} & w_{N-m 1} & \cdots & w_{N-m N} & ; & g_{N} \\
u_{00} & u_{01} & \cdots & u_{0 N} & ; & \vartheta_{0} \\
u_{10} & u_{11} & \cdots & u_{1 N} & ; & \vartheta_{1} \\
\vdots & \vdots & \cdots & \vdots & \vdots & \vdots \\
u_{m-10} & u_{m-11} & \cdots & u_{m-1 N} & ; & \vartheta_{m-1}
\end{array}\right]
$$


or the matrix equation

$$
\widetilde{W} A=\tilde{G}
$$

If $\operatorname{det}(\widetilde{W}) \neq 0$ can rewrite (16) in the form $A=\widetilde{W}^{-1} \tilde{G}$ and the $\mathrm{A}$ is uniquely determined. Thus the $m$ th order linear complex differential equation with variable coefficients (1) under the conditions (2) has an approximated solution. This solution is given by the Gegenbauer series (3). Also we can easily check the accuracy of the obtained solutions. Since the Gegenbauer series (3) is an approximate solution of (1), when the solutions $f(z)$ and its derivatives are substituted in (1), the resulting equation must be satisfied approximately; that is, for $z=z_{j} \in D, j=0,1,2, \ldots$

$$
E\left(z_{j}\right)=\left|\sum_{n=0}^{m} P_{n}\left(z_{j}\right) f^{(n)}\left(z_{j}\right)-g\left(z_{j}\right)\right| \cong 0
$$

or

$E\left(z_{j}\right) \leq 10^{-k_{j}} \quad\left(k_{j}\right.$ is any positive integer $)$

If $\max 10^{-k_{j}}=10^{-k} \quad$ ( $\mathrm{k}$ positive integer) is prescribed, then the truncation limit $\mathrm{N}$ is increased until the values $E\left(z_{j}\right)$ at each of the points $Z_{j}$ becomes smaller than the prescribed $10^{-k}$.

\section{Conclusion}

High-order linear complex differential equations are usually difficult to solve analytically. Then, it is required to obtain the approximate solutions. For this reason, a new technique using the Gegenbauer polynomials to solve numerically such equations is proposed. This method is based on computing the coefficients in the Gegenbauer series expansion of the solution of a linear complex differential equation. And the solutions are valid when the functions $P_{n}(z)$ and $g(z)$ are defined in the circular domain

\section{References}

[1] M. Çetin, M. Sezer and Ç. Güler, Lucas polynomial approach for system of high-order linear differential equations and residual error estimation. Mathematical problems in engineering, (2015), 625984.

[2] M. Sezer and S. Yüzbaşı, A collocation method to solve higher order linear complex differential equations in rectangular domains, Numerical Methods for Partial Differential Equations, (2010), 26:596-611.

[3] S. Yüzbaşı, M. Aynıül, and M. Sezer, A collocation method using Hermite polynomials for approximate solution of pantograph equations, Journal of the Franklin Institute. (2011),348:1128-1139.

[4] F. Düşünceli and E. Çelik, An Effective Tool: Numerical Solutions by Legendre 
Polynomials for High-order Linear Complex Differential Equations. British Journal of Applied Science \& Technology, (2015), 8(4): 348-355.

[5] M. Bagherpoorfard, and F.A. Ghassabzade, Hermite Matrix Polynomial Collocation Method for Linear Complex Differential Equations and Some Comparisons. Journal of Applied Mathematics and Physics. (2013), 1:58-64.

[6] S. Yüzbası, N. Şahin and M. Gülsu, A collocation approach for solving a class of complex differential equations in elliptic domains, Journal of Numerical Mathematics, (2011), 19: 225-246.

[7] Hochstrasser, U. W. "Orthogonal Polynomials." Handbook of Mathematical Functions with Formulas, Graphs, and Mathematical Tables. (M. Abramowitz and I. A. Stegun, eds.). New York: Dover, 1972. 